\title{
Detection of a Target Moving in a Network
}

\author{
J.-P. Le Cadre \\ IRISA/CNRS \\ Campus de Beaulieu \\ 35042, Rennes, \\ France. \\ lecadre@irisa.fr
}

\begin{abstract}
The area of search theory can be divided broadly on two parts, one-sided search and two-sided [7]. Thus, in this paper, we deal with a two-sided search games played by a searcher and a mobile target with a rather simple type of motion called the conditionally deterministic motion (CDM). Here, the target motion takes place on a network and, more precisely, on a set of possible paths.
\end{abstract}

After a general introduction of two-person zero-sum games, we examine various formulations of a search game for a target moving across a network. Then, this approach is extended to interdiction games and multiple detections.

Keywords: Detection, network, moving target, search games, resource management.

\section{Introduction}

Search theory is the discipline that treats the problem of how best to search for an object when the amount of searching efforts is limited and only probabilities of the object's possible position are given. A search (theory) problem is characterized by three pieces of data[7]: (i) the probabilities of the searched object (the "target") being in various possible locations; (ii) the local detection probability that a particular amount of local search effort could detect the target; (iii) the total amount of searching effort available. The problem is to find the optimal distribution of this total effort that maximizes the probability of detection[7].

The growth of the search theory literature has been chronicled in [3]. For instance, the last item (search games) is the primary focus of recent researches, including numerous sub-domains such as : mobile evaders, avoiding target, ambush games, inspection games and tactical games. For moving target problems, decisive progress have been made in developing search strategies that maximize the probability of detecting the (moving) target within a fixed amount of time. However, although the general formalism of search theory will be used subsequently, we shall study radically different problems.

The area of search theory can be divided broadly in two parts, one-sided search and two-sided[2],[1]. Even if Markovianity is a common assumption for modelling target motion, it is not so realistic for many situations. To a large extend, this is adapted to our ignorance about the target behavior. However, for many situations, we can have a more precise description of the target possibilities[2],[1]. Thus, in this paper, we deal with a two-sided search games played by a searcher and a mobile target with a rather simple type of motion called the conditionally deterministic motion (CDM). Here, the target motion takes place on a network and, more precisely, on a set of possible paths.

After a general introduction of two-person zero-sum games, we examine various formulations of a search game for a target moving across a network [5], [4]. Then, this approach is extended to interdiction games [8] and multiple detections [5].

\section{Search Games}

\subsection{Two-Person Zero Sum Games}

Games are the natural framework for avoiding the need of a strong prior about the target location; i.e. both target and searcher have (randomized) strategies. In this setting, we denote $a_{i j}$ the cost for player 1 to choose row $i$ while player 2 chooses column $j$. A two-person zero-sum game (denoted tpzg for the sequel) is a matrix game. If in a matrix game $A=\left(a_{i j}\right), i=1 \ldots . m, j=1 \ldots n$, there exists a couple $\left(i^{*}, j^{*}\right)$ s.t. $\forall i$ and $\forall j$, we have $a_{i j^{*}} \leq a_{i^{*} j^{*}} \leq a_{i^{*} j}$, then the couple $\left(i^{*}, j^{*}\right)$ is a saddle point for a pure strategy and we have:

$$
\max _{i} \min _{j} a_{i j}=\min _{j} \max _{i} a_{i j}=a_{i^{*} j^{*}}
$$


A mixed strategy for the player 1 is a $m$-uple denoted $x=\left(x_{1}, x_{2}, \ldots, x_{m}\right)$ where the $\left\{x_{i}\right\}$ are positive and sum to 1 . A mixed strategy for the player 2 is a $n$-uple denoted $y=\left(y_{1}, y_{2}, \ldots, y_{n}\right)$ with $y_{j} \geq 0 \forall j$ and $\sum_{j} y_{j}=1$. The meaning of the $\mathbf{x}$ vector is: player 1 chooses the pure strategy $i$ with the probability $x_{i}$. Similarly, the player 2 chooses the pure strategy $j$ with the probability $y_{j}$; total cost being $\sum_{i=1}^{m} \sum_{j=1}^{n} x_{i} y_{j} a_{i j}=\mathbf{x}^{T} A \mathbf{y}$. It has been proven (Von Neuman) that all the matrix games have a saddle point for mixed strategies; i.e. there always exists vectors $\mathbf{x}$ and $\mathbf{y}$ such that:

$$
\begin{aligned}
& \max _{\mathbf{x}} \min _{\mathbf{y}} \mathbf{x}^{T} A \mathbf{y}=\min _{y} \max _{x} \mathbf{x}^{T} A \mathbf{y}=\left(\mathbf{x}^{*}\right)^{T} A \mathbf{y}^{*}=v, \\
& x_{i} \geq 0 \text { and } \sum_{i} x_{i}=1 ; x_{i} \geq 0 \text { and } \sum_{j} y_{j}=1
\end{aligned}
$$

where $v$ is called the game value. Equivalently, a solution $\left\{\mathbf{x}^{*}, \mathbf{y}^{*}\right\}$ is characterized by:

$$
\begin{aligned}
& \sum_{i} a_{i j} x_{i}^{*} \geq v \quad j=1 \ldots \\
& \sum_{j} a_{i j} y_{j}^{*} \leq v \quad i=1 \ldots .
\end{aligned}
$$

The first condition sets that $\mathbf{x}^{*}$ assures at least $v$ whatever the pure straregy of player 2 is; idem for the second one. Every matrix game can be described (and solved) by the following linear optimization problem (player 1, primal problem) :

$$
\left\{\begin{array}{l}
\max _{\left(x_{1}, \ldots, x_{m}, x_{0}\right)} x_{0}, \\
\text { with the constraints: } \\
\sum_{i=1}^{m} x_{i} a_{i j}-x_{0} \geq 0 \quad j=1 \ldots n, \\
\sum_{i=1}^{m} x_{i}=1, \\
x_{i} \geq 0 \quad i=1 \ldots m .
\end{array}\right.
$$

The dual of this linear programming problem is the player 2 point of view, i.e.:

$$
\left\{\begin{array}{c}
\min _{\left(y_{1}, \ldots, y_{n}, y_{0}\right)} y_{0}, \\
\text { such that: } \\
\sum_{j=1}^{n} a_{i j} y_{j}-y_{0} \leq 0 \quad i=1 \ldots m \\
\sum_{j=1}^{n} y_{j}=1, \\
y_{j} \geq 0 \quad j=1 \ldots n .
\end{array}\right.
$$

Both problems give the same value $v$.

\subsection{A search game for a target moving across a network}

\subsubsection{Simple constraints}

Here, we are dealing with the detection of a target whose motion is constrained to be a path in a network.
The space of possible target positions is made of cells indexed by $j ; j=1,2, \ldots, m$. Time is also discretized in $n$ periods. A target path $\omega=\{j(t), t=1,2, \ldots, n\}$ is defined as a sequence of cell indexes. Thus, $j(t)$ is the cell occupied by the target at period $t$. The set of possible paths is known from the searcher. The target can choose any feasible path in the network. On another hand, the search effort is bounded above at each period. So, the aim of the searcher is to maximize the probability of detecting the target within the search constraints; while for the target it is to minimize the probability to be detected [6].

The number $K$ of possible paths is assumed to be finite. Let $T_{t}(\omega)$ the cell where the target is at period $t$ and the path $\omega$ having been selected by the target. Furthermore, if the target remains undetected at time $n$, it is the winner of the game. Here, the total search effort $\{C(t) \quad t=1,2, \ldots, n\}$ is indefinetely divisible. The search effort allocated to cell $j$ at time $t$ is denoted $\varphi(j, t)$. If the target is in the cell $j$ at time $t$, the conditional probability of detection is given by

$$
f(j, \varphi(j, t))=1-\exp [-\alpha(j) \varphi(j, t)],
$$

where $\alpha(j)$ is the visibility coefficient. The search policy $\Phi$ is defined by the spatio-temporal search efforts, i.e. $\Phi=\{\varphi(j, t)\}_{j, t}$. The parameters $\{C(t)\},\{\alpha(j)\}$ and the set of paths $\{\omega \in \Omega\}$ are known at the beginning of the game. Then, we denote $g(\omega, \Phi)$ the non-detection probability when the target uses the path $\omega$ (target) and the search efforts $\Phi$ (searcher) are in use :

$$
g(\omega, \Phi)=\exp \left[-\sum_{t} \alpha\left(T_{t}(\omega)\right) \varphi\left(T_{t}(\omega), t\right)\right] .
$$

The function $g(\omega, \Phi)$ is strictly convex w.r.t. the $\Phi$ components $\left(\Phi=\{\varphi(j, t)\}_{j, t}\right)$. Another fundamental point is that the search efforts $\varphi\left(T_{t}(\omega), t\right)$ act separately. Therefore, the optimal search strategy is unique and it is a pure strategy. Now, we define the mixed target strategy by $\mathbf{P}=\left\{p_{\omega}: \omega \in \Omega\right\}$, so that we have to consider the functional $G(\mathbf{P}, \Phi)$ defined as :

$$
G(\mathbf{P}, \Phi)=\sum_{\omega \in \Omega} p_{\omega} g(\omega, \Phi),
$$

and the optimization problem we have to deal with is :

$$
\left\{\begin{array}{l}
\underset{\mathbf{\Phi}}{\min } \max _{\mathbf{P}} g(\mathbf{P}, \Phi)=\max _{\mathbf{P}} \min _{\mathbf{\Phi}} g(\mathbf{P}, \Phi)=v, \\
\text { under the constraints : } \\
\sum_{j} \varphi(j, t) \leq C(t) \forall t \text { and } \varphi(j, t) \geq 0, \forall t \forall j \in E, \\
\sum_{\omega}^{j} p_{\omega}=1 \text { and } p_{\omega} \geq 0 \forall \omega \in \Omega .
\end{array}\right.
$$

${ }^{1}$ This density is arbitrary but motivated by operational considerations. Furthermore, it can be replaced by any concave or pseudo-concave functional 
Let $P^{*}=\left\{p_{\omega}^{*}\right\}$ and (resp.) $\Phi^{*}=\left\{\varphi^{*}(j, t)\right\}$ be the optimal strategies of the target and (resp.) the searcher and denote $\mu_{0}=\max _{\omega \in \Omega} g\left(\omega, \Phi^{*}\right)$. Then the following necessary conditions are straightforwardly deduced from the Karush-Kuhn-Tucker (denoted KKT) optimality conditions (see Appendix A and [6]):

$$
\begin{aligned}
& \text { Optimization of the search efforts: } \\
& \text { If } p_{\omega}^{*}>0 \text { then } \exp \left(-\sum_{t} \alpha\left(T_{t}(\omega)\right) \varphi^{*}\left(T_{t}(\omega), t\right)\right)=\mu_{0}, \\
& \text { If } p_{\omega}^{*}=0 \text { then } \exp \left(-\sum_{t} \alpha\left(T_{t}(\omega)\right) \varphi^{*}\left(T_{t}(\omega), t\right)\right) \leq \mu_{0}
\end{aligned}
$$$$
\text { Optimal (mixed) target strategy : }
$$$$
\begin{aligned}
& \text { If } \varphi^{*}(j, t)>0 \text { then } \alpha(j) \sum_{\omega \in \Omega(j, t)} p_{\omega}^{*}=\frac{\lambda_{t}}{\mu_{0}} \\
& \text { If } \varphi^{*}(j, t)=0 \text { then } \alpha(j) \sum_{\omega \in \Omega(j, t)} p_{\omega}^{*} \leq \frac{\lambda_{t}}{\mu_{0}}
\end{aligned}
$$

where $\Omega(j, t)=\left\{\begin{array}{ll}\omega & T_{t}(\omega)=j\end{array}\right\}$ and $\lambda_{t}$ is a Lagrange multiplier and while the game value is $G=\mu_{0}$. We remark that $\mu_{0}$ can be also characterized by :

$\mu_{0}=\min _{\varphi}\left\{\mu \quad: \sum_{t} \alpha\left(T_{t}(\omega)\right) \varphi\left(T_{t}(\omega), t\right) \geq-\log (\mu) \quad, \forall \omega \in \Omega\right\}$

which has the definite advantage to be linear w.r.t. the optimization parameters. So, denoting $z=-\log (\mu)$, the optimal search strategy can be obtained as the solution of the following linear programming LP5 problem :

$$
\left\{\begin{array}{l}
\text { maximize } z \\
\text { under the constraints: } \\
\sum_{t} \alpha\left(T_{t}(\omega)\right) \varphi\left(T_{t}(\omega)\right)-z \geq 0 \quad \forall \omega \in \Omega \\
\sum_{j} \varphi(j, t) \leq c_{t} \quad \forall t \\
\varphi(j, t) \geq 0 \quad \forall j \quad \forall t
\end{array}\right.
$$

Thus the searcher strategy can be efficiently obtained by solving LP5 w.r.t. $\left[z ;\{\varphi(j, t)\}_{j, t}\right]$, by means of the Simplex algorithm. The target strategy can be obtained as the solution of a linear system derived from 8 and 9. Examples will be provided later.

\section{Some examples}

Here a simple game with 3 cells $(m=3), 3$ periods $(n=3)$ and 4 paths $(\mathrm{K}=4)$ is considered. Paths are defined by : $\omega_{1}=(1,1,1), \omega_{2}=(1,2,2), \omega_{3}=(2,2,1), \omega_{4}=$ $(3,2,2)$.

The constraints on the temporal amounts of search effort are :

$C(1)=0.9, C(2)=0.3, C(3)=0.6$

while $\alpha(j)=1 \quad \forall j$.

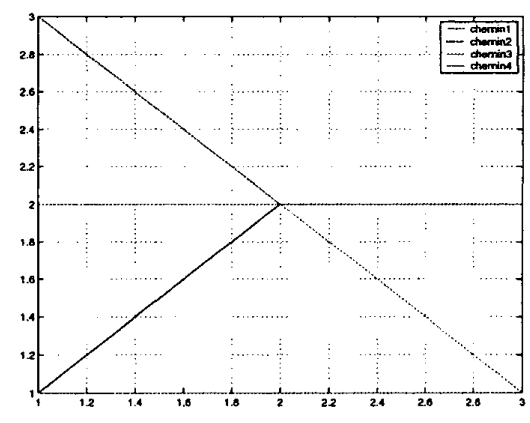

Figure 1: Elementary network

\begin{tabular}{|c|c|c|c|}
\hline & $t=1$ & $t=2$ & $t=3$ \\
\hline$j=1 \quad 0$ & 0.4000 & 0 & 0.4500 \\
\hline $\mathrm{j}=2 \quad 0$ & 0.1000 & 0.3000 & 0.1500 \\
\hline \begin{tabular}{l|l}
$j=3$ & 0
\end{tabular} & 0.4000 & 0 & 0 \\
\hline & & $\varphi^{*}(j, t)$ & \\
\hline$\omega_{1}$ & $\omega_{2}$ & $\omega_{3}$ & $\omega_{4}$ \\
\hline \multirow[t]{2}{*}{0.1667} & 0.1667 & 0.3333 & 0.3333 \\
\hline & & $p_{\omega}^{*}$ & \\
\hline
\end{tabular}

Applying the Simplex algorithm to LP5 we obtain a game value $\mu_{0}=0.4274$. The searcher and target strategies are given by the following tables :

We remark that the searcher concentrates search efforts on the target path crossing points. Let us now detail the determination of the target strategy. From the KKT conditions we deduce :

$$
\begin{cases}p_{\omega_{1}}+p_{\omega_{2}}-\lambda_{1} / \mu=0 & \text { for }: j=1, t=1 \\ p_{\omega_{1}}+p_{\omega_{3}}-\lambda_{3} / \mu=0 & \text { for }: j=1, t=3 \\ p_{\omega_{3}}-\lambda_{1} / \mu=0 & \text { for }: j=2, t=1 \\ p_{\omega_{2}}+p_{\omega_{3}}+p_{\omega_{4}}-\lambda_{2} / \mu=0 & \text { for }: j=2, t=2 \\ p_{\omega_{2}}+p_{\omega_{4}}-\lambda_{3} / \mu=0 & \text { for }:, j=2 t=3 \\ p_{\omega_{4}}-\lambda_{1} / \mu=0 & \text { for }: j=3, t=1 \\ p_{\omega_{1}}+p_{\omega_{2}}+p_{\omega_{3}}+p_{\omega_{4}}=1 . & \end{cases}
$$

thus the target strategy is obtained by solving the above linear system.

We consider now a more complicated game: i.e. 4 cells $(m=4), 6$ periods $(n=6)$ and 5 paths $(K=5)$ : 
$\omega_{1}=(3,4,4,3,4,3), \quad \omega_{2}=(3,3,3,4,3,3)$,
$\omega_{3}=(3,2,2,3,2,3), \quad \omega_{4}=(3,2,1,1,2,3)$, $\omega_{5}=(3,4,3,2,3,3)$.

Again we assume that $\alpha(j)=1 \quad \forall j$ and that the following constraints hold $C(1)=0.8, C(2)=0.7, C(3)=0.9$, $C(4)=0.4, C(5)=0.6$ et $C(6)=0.5$.

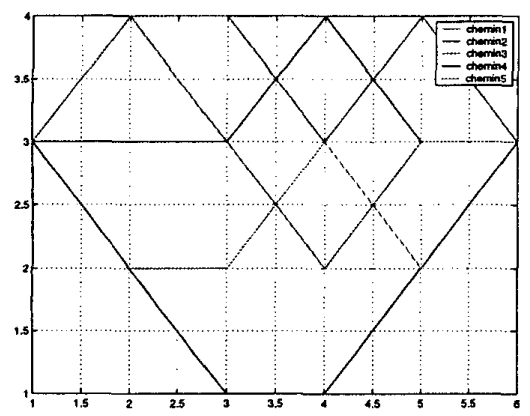

Figure 2: The network

The game value is 0.2307 and searcher and target strategies are given by the following tables :

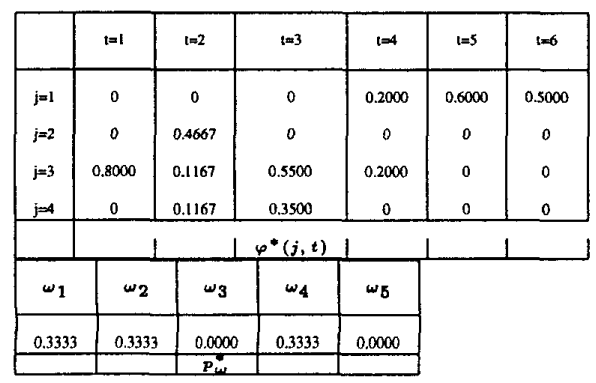

\subsubsection{Generalized constraints}

In the previous problem, the amount of search effort at period $t$ is bounded above by $C(t)$. However, three types of constraints denoted $\mathcal{C}_{i}$ have a natural interpretation in this context, namely:

$$
\left\{\begin{array}{l}
\mathcal{C}_{1}: \varphi(j, t) \leq B(j, t) \quad \forall t \quad \forall j \\
\mathcal{C}_{2}: \sum_{j} \varphi(j, t) \leq C(t) \quad \forall t \\
\mathcal{C}_{3}: \sum_{t} \sum_{j} \varphi(j, t) \leq D
\end{array}\right.
$$

Note that this problem has a specific meaning only if $\sum_{t} C(t)>D$ and again can be solved by means of the following linear programming algorithm LP6 :

$$
\left\{\begin{array}{l}
\operatorname{maximize} \quad z \\
\text { under the constraints : } \\
\sum_{t} \alpha\left(T_{t}(\omega)\right) \varphi\left(T_{t}(\omega)\right)-z \geq 0 \quad \forall \omega \in \Omega, \\
\sum_{j} \varphi(j, t) \leq c_{t} \quad \forall t \\
\sum_{t} \sum_{j} \varphi(j, t) \leq D \\
\varphi(j, t) \geq 0 \quad \forall j \quad \forall t .
\end{array}\right.
$$

Results

We consider again the simple game with 3 cells $(m=3), 3$ periods $(\mathrm{n}=3)$ and 4 paths $(\mathrm{K}=4)$ :

$\omega_{1}=(1,1,1), \omega_{2}=(1,2,2), \omega_{3}=(2,2,1), \omega_{4}=$ $(3,2,2)(\alpha(j)=1 \quad \forall j)$. The cell constraints are $C(1)=$ $0.9, C(2)=0.3, C(3)=0.6$, while the following contraint is added $\sum_{t} \sum_{j} \varphi(j, t) \leq 1.5$. The game value is 0.4066 and the optimal search and target strategies are :

\begin{tabular}{|c|c|c|c|}
\hline$t$ & 1 & 2 & 3 \\
\hline$j=1$ & 0.3000 & 0 & 0.4500 \\
$j=2$ & 0 & 0.3000 & 0.1500 \\
$j=3$ & 0.3000 & 0 & 0 \\
\hline$\omega_{1}$ & $\omega_{2}$ & $\omega_{3}$ & $\omega_{4}$ \\
\hline $0.5-2 \mathrm{a}$ & $\mathrm{a}$ & $2 \mathrm{a}$ & $0.5-\mathrm{a}$ \\
\hline
\end{tabular}

\subsection{An interdiction game}

We consider here the following game [8]. Again, a target is moving on a network but this time the search effort is not indefinitely divisible. At each period the target is transiting from a node $s$ and to an adjacent node $t$. Simultaneously, the searcher selects one arc $k$ in the network and inspects this arc. If the target is passing throughout the arc $k$, then it is detected with a probability $p_{k}$. These detection probabilities are known both from the target and the searcher.

The aim of the searcher is to find the inspection strategy which maximizes the probability that the target be detected. Opposite, the target strategy is to minimize it. Therefore, this problem can be viewed as a tpzg[?].

Let us now present a general formulation for this problem. Let $G=(N, A)$, a network with $N$ denoting the set of nodes and $A$ the set of arcs. A path in $G$, starting at the 
node $i_{0}$ and arriving to the node $i_{m}$ is defined as a sequence of nodes and arcs of the form $i_{0},\left(i_{0}, i_{1}\right), i_{1},\left(i_{1}, i_{2}\right), \ldots$, $i_{m-1},\left(i_{m-1}, i_{m}\right), i_{m}$. A path $l \in L$ is characterized by the arcs $A(l)$ composing it. The matrix $D$ is the incidence matrix: i.e. $d_{k, l}=1$ iff the path $l$ includes the arc $k$ and is equal to 0 else. The $l$-th column of the $D$ matrix (denoted $d(l))$ is the incidence vector for the path $l$.

Our aim is to solve a tpzg $Q$ where the pure strategy of the searcher is to select a path $l$, from $s$ to $t$. Let us define the $z$ vector by : $z_{k}=1$ if the searcher inspects the arc $k$ and $z_{k}=0$ else. Then, the cost function $V$ for $Q$ is defined by : $V(z, l)=\sum_{k \in A(l)} p_{k} z_{k}$, which is the probability that the target be detected. The $V(z, l)$ expectation is denoted $\psi$ and is the interdiction probability. For the searcher, the objective is to maximize $\psi$, while it is the converse for the target.

Let $x_{k}$ be the probability that the searcher inspects the $k$ arc and denote $y_{l}$ the probability that the target chooses the path $l$. Thus, the vectors $\mathbf{x}$ and (resp.) y represent the mixed strategies of the searcher, (resp.) target; and :

$$
\psi=E(V(z, l))=\sum_{k \in A} \sum_{l \in L} x_{k} p_{k} d_{k l} \cdot y_{l}=\mathbf{x} P D \mathbf{y}
$$

The optimization problem can be written as the matrix game [8] Maxmin0 :

$$
\left\{\begin{array}{c}
\max _{\mathbf{x}} \min _{\mathbf{y}} \mathbf{x} P D \mathbf{y}, \\
\text { subject to: } \\
\sum_{i=1}^{k} x_{i}=1 \quad \sum_{j=1}^{l} y_{j}=1, \\
x_{i} \geq 0 \quad \forall i, \quad y_{j} \geq 0 \quad \forall j .
\end{array}\right.
$$

$Q$ being a finite matrix game can be solved by the following linear programm :

$$
\left\{\begin{array}{c}
\nu^{*}=\min _{(\mathbf{y}, \nu)} \nu \\
\text { under the constraints: } \\
P D \mathbf{y}-\mathbf{1} \nu \leq \mathbf{0}, \\
\sum_{j=1}^{l} y_{j}=1 \quad y_{j} \geq 0 \quad \forall j .
\end{array}\right.
$$

where all the terms of the $1 \nu$ vector are equal to $\nu$. If the above problem gives us the target strategy, the searcher strategy is obtained by dualization, i.e. :

$$
\left\{\begin{array}{c}
\nu^{*}=\max _{(\mathbf{x}, \nu)} \nu \\
\text { under the constraints : }(P D)^{T} \mathbf{x}-1 \nu \leq \mathbf{0}, \\
\sum_{i=1}^{k} x_{i}=1 \quad x_{i} \geq 0 \quad \forall i
\end{array}\right.
$$

Not surprisingly, the problem may be solved by a Simplexlike algorithm. However, a major problem may be the cardinality of the set of paths which can grows very rapidly.

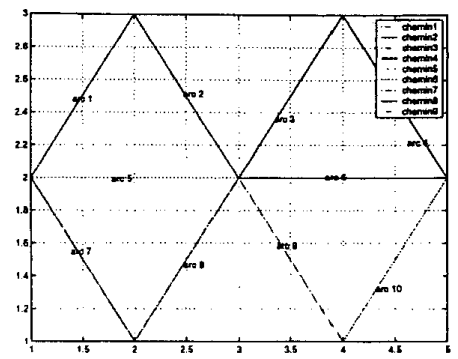

Figure 3: The network

\section{Results}

The network as well as the possible paths are described below :

$$
\begin{aligned}
& \text { Path 1: } l_{1}=\{1,2,3,4\} \text {. } \\
& \text { Path 3: } l_{3}=\{1,2,9,10\} \\
& \text { Path 4: } i_{4}=\{5,3,4\} \\
& \text { Path } 6: i_{6}=\{5,9,10\} \\
& \text { Path 7: } l_{7}=(7,8,3,4) \text {, } \\
& \begin{array}{ll}
\text { Path } 8: & \left.l_{8}=\{7,8,6\}\right) \\
\text { Path } \theta: & l_{\theta}=\{7,8,9,10\}
\end{array} \\
& D=\left(\begin{array}{l}
1 \\
1 \\
1 \\
0 \\
0 \\
0 \\
0 \\
0 \\
0
\end{array}\right. \\
& \begin{array}{llll}
1 & 1 & 1 & 1 \\
1 & 1 & 0 & 0 \\
1 & 1 & 0 & 0 \\
0 & 0 & 1 & 1 \\
0 & 0 & 0 & 0 \\
0 & 0 & 0 & 0 \\
0 & 0 & 1 & 1 \\
0 & 0 & 0 & 0 \\
0 & 0 & 0 & 0
\end{array} \\
& \begin{array}{llll}
1 & 0 & 0 & 0 \\
0 & 0 & 1 & 0 \\
0 & 0 & 0 & 0 \\
1 & 1 & 0 & 0 \\
0 & 1 & 1 & 0 \\
0 & 1 & 0 & 0 \\
1 & 0 & 0 & 1 \\
0 & 0 & 1 & 1 \\
0 & 0 & 0 & 1
\end{array} \\
& \left.\begin{array}{lll}
0 & 0 & 0 \\
0 & 0 & 0 \\
0 & 1 & 1 \\
0 & 0 & 0 \\
0 & 0 & 0 \\
0 & 1 & 1 \\
1 & 0 & 0 \\
1 & 0 & 0 \\
1 & 1 & 1
\end{array}\right)
\end{aligned}
$$

\subsection{Optimal search for gain maximization}

In the previous problems, we were considering both target and searcher strategies in a game perspective. This is no longer the case, even if the general context is still the search of a target moving in a network. Here, the aim of the searcher is to detect as frequently as possible the target. Thus, the objective functional is no longer binary. The interest of this approach is that it is closer to the objective of target tracking. Let us present now the modelling [5].

Let $G(V, A)$ be this network with $V$ the set of nodes and $A$ the set of arcs $(n=\# A)$. The target has available a set $\Omega$ of possible paths and selects one of them for all the search duration. The path $l \in \Omega$ is made of $m_{l}$ arcs denoted $l(1), l(2), \ldots, l\left(m_{l}\right)$. The probability that the target selects the path $l$ for transiting across the network is equal to $\pi(l) \geq 0\left(\sum_{l \in \Omega} \pi(l)=1\right)$.

A search plan is made of elementary efforts $\varphi_{k} \geq 0$ distributed on the arcs and denoted $\phi=\left\{\varphi_{1}, \ldots, \varphi_{n}\right\}$. Conditionnally to the search effort $\varphi_{k}$ (on the arc $k$ ) and to the event "target is passing throughout this arc", it is detected with a probability $p_{k}=1-\exp \left(-\alpha_{k} \varphi_{k}\right)\left(\alpha_{k} \geq 0\right)$. If the 
target is detected on the $\operatorname{arc} k$, the searcher has an income of $V_{k} \geq 0$, at an expense equal to $C_{k} \geq 0$. The objective functional is the gain of the search, i.e. incomes minus expenses. For instance, we can assume that the probability $P(l, i)$ that the target be detected on one the arcs $l(1), l(2), \ldots, l(i)$ conditionally to the event "target is passing throughout the path $l \in \Omega$ " is ${ }^{2}$ :

$$
P(l, i)=1-\exp \left[-\sum_{j=1}^{i} \alpha_{l(j)} \varphi_{l(j)}\right]
$$

Thus, the probability to detect on the $\operatorname{arc} l(i)$ is $P(l, i)-$ $P(l, i-1)$, with $P(l, 0)=0$; with an income equal to $V_{l(i)}$. Then, the objective functional is the gain $R(\phi)$ defined by :



Figure 4: The network

$$
\begin{aligned}
R(\phi)= & \sum_{l \in \Omega} \pi(l) \sum_{i=1}^{m_{l}} V_{l(i)}(P(l, i)-P(l, i-1))-\sum_{k=1}^{n} C_{k} \phi_{k}, \\
\text { or : } & \begin{aligned}
R(\phi)= & \sum_{l \in \Omega_{2}} \pi(l)\left\{V_{l\left(m_{l}\right)} P\left(l, m_{l}\right)+\sum_{i=1}^{m_{l}-1}\left(V_{l(i)}-V_{l(i-1)}\right) P(l, i)\right\} \\
& -\sum_{k=1}^{n} C_{k} \phi_{k} .
\end{aligned}
\end{aligned}
$$

It is strictly concave w.r.t. $\phi$ and the problem is to maximize a concave functional on a convex set. Therefore, the solution is unique, i.e. $\max _{\varphi} R(\varphi)$ with the usula constraints $\sum_{k=1}^{n} \varphi_{k} \leq \mathcal{C}$. Again, it can be solved by linear programming algorithms.

\section{Results}

We consider the network illustrated by fig. 4 . The target has 5 paths available and the total amount $(\mathcal{C})$ of search effort is equal to 5 . This total amount is splitted everywhere on the 12 arcs. Possible paths and probabilities that the target takes a given path are :

Path $1: l_{1}=\{1,2,3\} \pi\left(l_{1}\right)=1 / 5$,

Path $2: l_{2}=\{1,7,8,3\} \pi\left(l_{2}\right)=1 / 5$,

Path $3: l_{3}=\{4,5,6\} \pi\left(l_{3}\right)=1 / 5$,

Path $4: l_{4}=\{4,9,10,6\} \pi\left(l_{4}\right)=1 / 5$,

Path $5: l_{5}=\{11,12\} \pi\left(l_{5}\right)=1 / 5$.

${ }^{2}$ In this formulation of $P(l, i)$, we assume independence of elementary detections, a quite criticable hypothesis

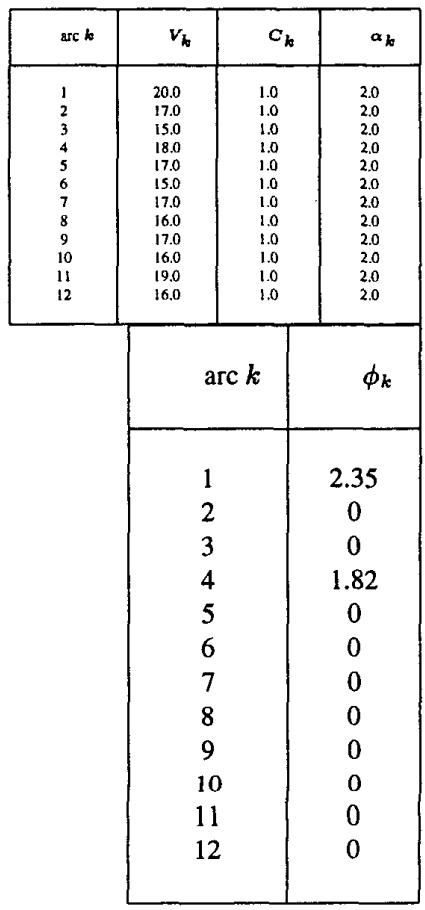

\subsection{Dynamic search game}

Here, we restrict to two cells and the evader begins at cell 1. The evader must choose a period $\sigma \in\{1, \ldots, T-1\}$ for going from cell 1 to cell 2 and then another period $\tau\{\sigma+1, \ldots, T\}$ for going from cell 2 to the evader-target. Let $z_{t}$ the search effort allocated to cell 1 , the rest being allocated to cell 2 . Assume, furthermore, that the elementary non-detection probability is exponential; then the non-detection probability for period $t\left(P_{n d}(t)\right)$ is obtained by considering the following events (see [9] for all the definitions):

1. evader remains in cell 1 , throughout the whole scenariol $(1 \leq t<\sigma), \rightarrow P_{n d}=\beta_{1}^{z_{t}}$

2. evader is in cell 1 and moves to cell $2,(t=\sigma), \rightarrow P_{n d}=$ $\alpha_{1}^{z_{t}}$

3. evader remains in cell $2(\sigma<t<\tau), \rightarrow P_{n d}=\beta_{2}^{1-z_{t}}$ 
4. evader in cell 2 and moves to the target $(\sigma<t<\tau)$, $\rightarrow$ $P_{n d}=\alpha_{2}^{1-z_{t}}$.

We also assume that $\left(\alpha_{i}<\beta_{i} \leq 1\right)$ and we have to consider a tpzg where the evader strategy (player 1 ) is defined by the two transition numbers $\sigma$ and $\tau$, while the searcher strategy is defined by the $T$-uple $\mathrm{z}=\left\{z_{1}, \cdots, z_{T}\right\}$. The objective functional stands as follows:

$$
P(\sigma, \tau, \mathrm{z})=\left[\prod_{t=1}^{\sigma-1} \beta_{1}^{z_{t}}\right] \alpha_{1}^{z_{\sigma}} \quad\left[\prod_{t=\sigma+1}^{\tau-1} \beta_{2}^{1-z_{t}}\right] \alpha_{2}^{1-z_{\tau}} .
$$

Moreover, the following assuptions are usual:

- $z_{t+1} \leq z_{t}, t=1, \ldots, T-1$,

- the searcher strategy is indivisible, i.e. $z_{t} \in\{0,1\}$

A straightforward consequence of these two assumptions is that there exists a period $s$ for which:

$$
\left\{\begin{array}{c}
z_{t}=1 \text { for } t \leq s \\
z_{t}=0 \text { else. }
\end{array}\right.
$$

Thus, the searcher strategy is completely defined by $s$ and the objective functional becomes:

$$
P(\sigma, \tau, s)=\left\{\begin{array}{l}
\beta_{1}^{s} \beta_{2}^{\tau-\sigma-1} \alpha_{2} \text { if } \mathrm{s}<\sigma \\
\beta_{1}^{\sigma-1} \alpha_{1} \beta_{2}^{r-s-1} \alpha_{2} \text { if } \sigma \leq s<\tau, \\
\beta_{1}^{\sigma-1} \alpha_{1} \text { if } \mathrm{s} \geq \tau
\end{array}\right.
$$

The mixed evader strategy amounts to considering the probability to select the couple $\{\sigma, \tau\}$, while for the searcher it is to choose $s$ with a probability $v_{s}$. The searcher strategy $v_{s}$ is obtained by solving the following linear programm:

$$
\left\{\begin{array}{l}
\min \xi \\
\xi \geq \sum_{s=1}^{T-1} v_{s} P(\sigma, \tau, s), \quad 1 \leq \sigma<\tau \leq t \\
\sum_{s=1}^{T-1} v_{s}=1 \\
v_{s} \geq 0, \quad 1 \leq s \leq T-1 .
\end{array}\right.
$$

The evader and searcher strategies are made more precise by the following results [9]

$$
\begin{aligned}
& \text { Proposition 1. The optimal evader strategy [9] } \\
& \text { is such that } \tau=\sigma+1 \text { Thus, the }\left\{v_{s}\right\} \\
& \text { must minimize } \max _{1 \leq \sigma<\tau \leq T}\left[\sum_{s-1}^{T-1} v_{s} P(\sigma, \tau, s)\right]= \\
& \max _{1 \leq \sigma<T}\left[\sum_{s=1}^{\sigma-1} v_{s}\left(\beta_{1}^{s} \alpha_{2}\right)+v_{\sigma}\left(\beta_{1}^{\sigma-1} \alpha_{1} \alpha_{2}\right)+\sum_{s=\sigma+1}^{T-1} v_{s}\left(\beta_{1}^{\sigma-1} \alpha_{1}\right)\right] \\
& \text { we remark that the searcher has a clear "tendency" to choose } s \\
& \text { on the first steps. }
\end{aligned}
$$

Proposition 2. The optimal searcher strategy [9] is given by solving the following linear system:

$\left\{\begin{array}{l}\sum_{s=1}^{\sigma-1} v_{s}\left(\beta_{1}^{s} \alpha_{2}\right)+v_{\sigma}\left(\beta_{1}^{\sigma-1} \alpha_{1} \alpha_{2}\right)+\sum_{s=\sigma+1}^{T-1} v_{s}\left(\beta_{1}^{\sigma-1} \alpha_{1}\right)=\xi^{*} \\ \text { for: } 1 \leq \sigma \leq t-1, \text { and: } \\ \sum_{s=1}^{T-1} v_{s}=1\end{array}\right.$
Results Consider the following values for the non-detection functions: $\beta_{1}=0.9, \alpha_{1}=0.6, \alpha_{2}=0.5$, then we have for $t=10$ (game value 0.4214):

\begin{tabular}{|c|c|}
\hline$t$ & $v_{s}$ \\
\hline 1 & 0.5954 \\
2 & 0.2409 \\
3 & 0.0975 \\
4 & 0.0394 \\
5 & 0.0160 \\
6 & 0.0065 \\
7 & 0.0027 \\
$*$ & 0.0011 \\
9 & 0.0005 \\
\hline
\end{tabular}

\section{Conclusions}

Various formulations of search games for detecting a target transiting across a network have been considered. All of them share a general framework based on tpzg and linear programming. It is worth to mention that linear programming permits to consider a large number of paths. However, to take benefit from this great tool, the objective functional must be separable which may be questionable.

\section{A Appendix A}

More generally, we consider the functional $G(\mathbf{P}, \Phi)$ defined by:

$$
G(\mathbf{P}, \Phi)=\sum_{\omega \Omega} p_{\omega} \prod_{t \in T} f\left[T_{t}(\omega), \varphi\left(T_{t}(\omega)\right)\right]
$$

where the functional $f$ stands for the non-detection probability. We have to deal with the following problem; find $\mathbf{P}^{*}$ (target) and $\Phi^{*}$ such that :

$$
G\left(\mathbf{P}, \Phi^{*}\right) \leq G\left(\mathbf{P}^{*}, \Phi^{*}\right) \leq G\left(\mathbf{P}^{*}, \Phi\right) \quad \forall \mathbf{P}, \forall \Phi .
$$

So, we have to examine two problems.

\section{A.1 First sub-problem} $G\left(\mathbf{P}, \Phi^{*}\right) \leq G\left(\mathbf{P}^{*}, \Phi^{*}\right)$.

We have to find the necessary conditions for the following problem :

$$
\mid \begin{aligned}
& \min -G\left(\mathbf{P}, \Phi^{*}\right) \\
& \sum_{\mathbf{P}} \text { such that : } \\
& \sum_{\omega \in \Omega} p_{\omega}=1, p_{\omega}>0 \forall \omega \in \Omega .
\end{aligned}
$$

The associated Lagrangean $\left(\nu_{\omega} \geq 0 \forall \omega \in \Omega\right)$ is :

$\mathcal{L}(\lambda, N)=-G\left(\mathbf{P}, \Phi^{*}\right)+\lambda\left(\sum_{\omega \in \Omega} p_{\omega}-1\right)-\sum_{\omega \in \Omega} \nu_{\omega} p_{\omega}$

So that we have two cases to consider: 
a)-First subcase: $p_{\omega}^{*}>0$;

$\mid \begin{aligned} & \mathrm{KKT} \rightarrow-\prod_{t \in T} f\left[T_{t}(\omega), \varphi^{*}\left(T_{t}(\omega)\right)\right]+\lambda=0, \\ & \text { or, denoting } P\left(\omega, \Phi^{*}\right)=\prod_{t \in T} f\left[T_{t}(\omega), \varphi^{*}\left(T_{t}(\omega)\right)\right], \\ & P\left(\omega, \Phi^{*}\right)=\mathrm{cst}, \text { if } ; \quad p_{\omega}^{*}>0, \forall \omega \in \Omega .\end{aligned}$

b)-Second subcase: $p_{\omega}^{*}=0$;

Then from the KKT conditions we deduce $-\prod_{t \in T} f\left[T_{t}(\omega), \varphi^{*}\left(T_{t}(\omega)\right)\right]+\lambda-\nu_{\omega}=0$.

Thus in the exponential case we obtain conditions (8).

\section{A.2 Second sub-problem}

The problem we have to face is :

$$
\mid \begin{aligned}
& \min _{\mathbf{\Phi}} G\left(\mathbf{P}^{*}, \Phi\right) \\
& \mathcal{C}: \sum_{j \in E} \varphi(j, t)=C_{t}, \varphi(j, t) \geq 0 .
\end{aligned}
$$

The Lagrangean then takes the following form :

$$
\begin{aligned}
\mathcal{L}(\Lambda) & =\sum_{j \in E}\left\{\sum_{\omega \in \Omega} p_{\omega}^{*} \prod_{t \neq t_{0}} f\left(\varphi\left(T_{t}(\omega)\right)\right)\right\} f\left(\varphi\left(j, t_{0}\right)\right) \text { (32) } \\
& +\Lambda^{T}\left(\sum_{t} \varphi(j, t)-C_{t}\right)_{t=1}^{t=T}+\operatorname{pos} .(\varphi(j, t)) .
\end{aligned}
$$

Denoting $G\left(j, t_{0}\right)=\left\{\sum_{\omega \in \Omega} p_{\omega}^{*} \prod_{t \neq t_{0}} f\left(\varphi\left(T_{t}(\omega)\right)\right)\right\}$ and assuming $\varphi^{*}\left(j, t_{0}\right)>0$, we have (KKT conditions) :

$$
G\left(j, t_{0}\right) f^{\prime}\left(\varphi^{*}\left(j, t_{0}\right)\right)+\lambda_{t_{0}}=0
$$

so that in the exponential case we have :

$$
\sum_{\omega \in \Omega\left(j, t_{0}\right)}\left\{p_{\omega}^{*} \prod_{t} f\left(\varphi^{*}\left(T_{t}(\omega)\right)\right\}=\frac{\lambda_{t_{0}}}{\omega_{j}}\right.
$$

where $\Omega\left(j, t_{0}\right)$ stands for the set of paths passing by the cell $j$, at time $t_{0}$.

Now, from the KKT conditions of the first sub-problem, we know that $\prod_{t} f\left(\varphi^{*}\left(T_{t}(\omega)\right)=\mathrm{cst}>0\right.$ if there exists $\omega \in \Omega\left(j, t_{0}\right)$ such that $p_{\omega}^{*}>0$. In this case, we thus have :

$$
\sum_{\omega \in \Pi\left(j, t_{0}\right)} p_{\omega}^{*}=\operatorname{cst}>0 \text {. }
$$

If this is not the case; i.e. if $p_{\omega}^{*}=0, \forall \omega \in \Omega\left(j, t_{0}\right)$ then :

$$
\sum_{t} \alpha\left(T_{t}(\omega)\right) \varphi^{*}\left(T_{t}(\omega)\right) \leq \mu_{0}, \forall \omega \in \Omega\left(j, t_{0}\right),
$$

so that, finally :

$$
\left\{\begin{array}{l}
\varphi^{*}\left(j, t_{0}\right) \rightarrow \sum_{\omega \in \Omega\left(j, t_{0}\right)} p_{\omega}^{*}=\lambda_{t_{0}} \\
\varphi^{*}\left(j, t_{0}\right) \rightarrow \sum_{\omega \in \Omega\left(j, t_{0}\right)} p_{\omega}^{*} \leq \lambda_{t_{0}}
\end{array}\right.
$$

\section{References}

[1] M. Sakaguchi, "A two-Sided resource Allocation Game in Search for a Stationary Object". Math. Japonica, vol. 32, n0. 6, pp. 979-991, 1987.

[2] L.C. Thomas and A.R. Washburn, "Dynamic Search Games". Operations Research, vol. 39, no. 3, pp 415-422, May-June 1991.

[3] S.J. Benkovski, M.G. Monticino and J.R. Weisinger, "A Survey of the Search Theory Literature". NAVAL RESEARCH LOGISTICS, vol.-38, pp. 469-491, 1991.

[4] R. Hohzaki, K. Iida, "A search game when a search path is given". European Journal of Operational Research 124, pp 114-124, 2000.

[5] R. Hohzaki, K. lida and M. Teramoto, "Optimal search for a moving target with no time information maximizing the expected reward". Journal of the operations research, society of Japan, Vol.42, no.2, 167-179, June 1999.

[6] K. lida, R. Hohzaki and S. Furui, "A search game for a mobile target with the conditionally deterministic motion defined by paths". Journal of the operations research, society of Japan, Vol.39, no.4, 501-511 , December 1996.

[7] L.D. Stone, Theory of Optimal Search, 2-nd ed. . Operations Research Society of America, ORSA Books, Arlington, VA, 1989.

[8] R.K. Wood, "Deterministic Network Interdiction". Mathl. Comput. Modelling, Vol. 17, no. 2, pp 1-18, 1993.

[9] T.J. Stewart, "A two-cell model of search for an evading target". European Journal of Operational Research, vol. 8, pp 369-378. 